\title{
Duality for a Control Problem Involving Support Functions
}

\author{
I. Husain1, Abdul Raoof Shah², Rishi K. Pandey ${ }^{1}$ \\ ${ }^{1}$ Department of Mathematics, Jaypee University of Engineering and Technology, Guna, India \\ ${ }^{2}$ Department of Statistics, University of Kashmir, Srinagar, India \\ Email: ihusain11@yahoo.com
}

Received 14 September 2014; revised 12 October 2014; accepted 8 November 2014

Copyright (C) 2014 by authors and Scientific Research Publishing Inc.

This work is licensed under the Creative Commons Attribution International License (CC BY).

http://creativecommons.org/licenses/by/4.0/

(c) (1) Open Access

\section{Abstract}

Mond-Weir type duality for control problem with support functions is investigated under generalized convexity conditions. Special cases are derived. A relationship between our results and those of nonlinear programming problem containing support functions is outlined.

\section{Keywords}

\section{Control Problem, Support Function, Generalize Convexity, Converse Duality, Nonlinear Programming}

\section{Introduction and Preliminaries}

Consider the following control problem containing support functions introduced by Husain et al. [1]

$$
\underset{(x, u)}{\operatorname{Minimize}} \int_{I}(f(t, x(t), u(t))+S(u(t) \mid K)) \mathrm{d} t
$$

subject to

$$
\begin{aligned}
& x(a)=0=x(b) \\
& g^{j}(t, x(t), u(t))+S\left(x(t) \mid C^{j}\right) \leq 0, t \in I, j=1,2, \cdots, m \\
& \dot{x}(t)=h(t, x(t), u(t)), t \in I
\end{aligned}
$$

where

1) $x: I \rightarrow R^{n}$ is a differentiable state vector function with its derivative $\dot{x}$ and $u: I \rightarrow R^{m}$ is a smooth control vector function. 
2) $R^{n}$ denotes an $n$-dimensional Euclidean space and $I=[a, b]$ is a real interval.

3) $f: I \times R^{n} \times R^{m} \rightarrow R, g: I \times R^{n} \times R^{m} \rightarrow R$ and $h: I \times R^{n} \times R^{m} \rightarrow R^{n}$ are continuously differentiable.

4) $s(x(t) \mid K)$ and $s\left(x(t) \mid C^{j}\right), \quad j=1,2, \cdots, m$ are the support function of the compact set $K$ and $C^{j}(j=1,2, \cdots, m)$ respectively.

Denote the partial derivatives of $f$ where by $f_{t}, f_{x}$ and $f_{t}$,

$$
f_{t}=\frac{\partial f}{\partial t}, f_{x}=\left[\frac{\partial f}{\partial x^{1}}, \frac{\partial f}{\partial x^{2}}, \cdots, \frac{\partial f}{\partial x^{n}}\right], f_{u}=\left[\frac{\partial f}{\partial u^{1}}, \frac{\partial f}{\partial u^{2}}, \cdots, \frac{\partial f}{\partial u^{m}}\right]
$$

where superscript denote the vector components. Similarly we have $h_{t}, h_{x}, h_{u}$ and $g_{t}, g_{x}, g_{u} . X$ is the space of continuously differentiable state functions $x: I \rightarrow R^{n}$. Such that $x(a)=0$ and $x(b)=0$ and are equipped with the norm $\|x\|=\|x\|_{\infty}+\left\|D_{x}\right\|_{\infty}$, and $U$, the space of piecewise continuous control vector functions $u: I \rightarrow R^{m}$ having the uniform norm \|\|$_{\infty}$. The differential Equation (2) with initial conditions expressed as

$x(t)=x(a)+\int_{a}^{b} h(s, x(s), y(s)) \mathrm{d} s t \in I$ may be written as $H=H(x, y)$, where

$H: X \times U \rightarrow C\left(I, R^{n}\right), C\left(I, R^{n}\right)$, being the space of continuous function from $I$ to $R^{n}$ defined as

$H(x, y)(t)=h(t, x(t), y(t))$. In the derivation of these optimality condition, some constraint qualification to make the equality constraint locally solvable [2] and hence the Fréchét derivative of $D x-H(x, u)=Q(x, u)$ (say) with respect to $(x, u)$, namely $Q^{\prime}=Q^{\prime}(x, u)=\left[D x-H_{x}(x, u),-H_{u}(x, u)\right]$ are required to be surjective. In [1], Husain et al. derived the following Fritz john type necessary optimality for the existence of optimal solution of (CP).

Proposition 1. (Fritz John Condition): If $(\bar{x}, \bar{u})$ is an optimal solution of (CP) and the Fréchét derivative $Q^{\prime}$ is surjective, then there exist Langrange multipliers $\alpha \in R$ and piecewise smooth $\lambda: I \rightarrow R^{m}, u: I \rightarrow R^{r}$, $z: I \rightarrow R^{m}$ and $\omega^{j}: I \rightarrow R^{n}$ such that for all $t$,

$$
\begin{gathered}
\alpha f_{x}(t, \bar{x}, \bar{u})+\sum_{j=1}^{m} \lambda^{j}(t)\left(g_{x}^{j}(t, \bar{x}, \bar{u})+\omega^{j}(t)\right)+\mu(t)^{\mathrm{T}} h_{x}(t, \bar{x}, \bar{u})+\dot{\mu}(t)=0, t \in I \\
\alpha f_{u}(t, \bar{x}, \bar{u})+\lambda^{\mathrm{T}}(t) g_{u}(t, \bar{x}, \bar{u})+\mu(t)^{\mathrm{T}} h_{u}(t, \bar{x}, \bar{u})=0, t \in I \\
\sum_{j=1}^{m} \lambda^{j}(t)\left(g_{x}^{j}(t, \bar{x}, \bar{u})+x^{\mathrm{T}}(t) \omega^{j}(t)\right)=0, t \in I \\
\mu^{\mathrm{T}}(t) z(t)=s(x(t) \mid K) \\
x(t) \omega^{j}(t)=s\left(x(t) \mid C^{j}\right), j=1,2, \cdots, m \\
z(t) \in K, \omega^{j}(t) \in C^{j}, j=1,2, \cdots, m \\
(\alpha, \lambda(t)) \geq 0, t \in I \\
(\alpha, \lambda(t), u(t)) \neq 0, t \in I
\end{gathered}
$$

As in [3], Husain et al. [1] pointed out if the optimal solution for (CP) is normal, then the Fritz john type optimal conditions reduce to the following Karush-Kuhn-Tucker optimal conditions.

Proposition 2. If $(\bar{x}, \bar{u})$ is an optimal solution and is normal and $Q^{\prime}$ is surjective, there exist piecewise smooth $\lambda: I \rightarrow R^{m}$ with $\lambda^{\mathrm{T}}=\left(\lambda_{1}, \lambda_{2}, \cdots, \lambda_{m}\right), u: I \rightarrow R^{n}, z: I \rightarrow R^{n}$ and $\omega^{j}: I \rightarrow R^{n}, \quad j=1,2, \cdots, m$ such that

$$
\begin{gathered}
f_{x}(t, \bar{x}, \bar{u})+\sum_{j=1}^{m} \lambda^{j}(t)\left(g_{x}^{j}(t, \bar{x}, \bar{u})+\omega^{j}(t)\right)+\mu(t)^{\mathrm{T}} h_{x}(t, \bar{x}, \bar{u})=\dot{\mu}(t) \\
f_{u}(t, \bar{x}, \bar{u})+z+\lambda(t)^{\mathrm{T}} g_{u}(t, \bar{x}, \bar{u})+\mu(t)^{\mathrm{T}} h_{u}(t, \bar{x}, \bar{u})=0, t \in I \\
\sum_{j=1}^{m} \lambda^{j}(t)\left(g_{x}^{j}(t, \bar{x}, \bar{u})+x^{\mathrm{T}}(t) \omega^{j}(t)\right)=0, t \in I
\end{gathered}
$$




$$
\begin{gathered}
u(t)^{\mathrm{T}} z(t)=s(x(t) \mid K) \\
x(t)^{\mathrm{T}} \omega^{j}(t)=s\left(x(t) \mid C^{j}\right), j=1,2, \cdots, m \\
\lambda^{j}(t) \geq 0, t \in I, j=1,2, \cdots, m \\
z(t) \in K, \omega^{j}(t) \in C^{j}, j=1,2, \cdots, m
\end{gathered}
$$

Using the Karush-Kuhn-Tucker type optimality condition given in Proposition 2, Husain et al. [1] presented the following Wolfe type dual to the control problem (CP) and proved usual duality theorem under the pseudoconvexity of $\int_{I}\left\{f(t, . .)+.() z.(t)+\sum_{i=1}^{m} \lambda^{i}(t)\left(g^{i}(t, . .)+.(.)^{\mathrm{T}} \omega^{i}(t)+\mu(t)^{\mathrm{T}}(t, . .)+.\dot{x}().\right)\right\} \mathrm{d} t$ for all $z(t) \in K$, and $\omega^{i}(t) \in C^{i}, \quad i=1,2, \cdots, m$.

(WCD): Maximize

subject to

$$
\int_{I}\left\{f(t, x, u)+\mu(t)^{\mathrm{T}} z(t)+\sum_{j=1}^{m} \lambda^{i}(t)\left(g^{j}(t, x, u)+x(t)^{\mathrm{T}} \omega^{j}(t)\right)+\mu(t)^{\mathrm{T}}(h(t, x, u)-\dot{x}(t))\right\} \mathrm{d} t
$$

$$
\begin{gathered}
x(a)=0, \alpha(b)=0 \\
f_{x}(t, x, u)+\sum_{j=1}^{m} \lambda^{i}(t)\left(g^{j}(t, x, u)+\omega^{j}(t)\right)+\mu(t)^{\mathrm{T}} h_{x}(t, x, u)+\dot{\mu}(t)=0, t \in I \\
f_{u}(t, x, u)+\lambda^{\mathrm{T}}(t) g(t, x, u)+\mu(t)^{\mathrm{T}} h_{u}(t, x, u)=0, t \in I \\
\lambda^{i}(t) \geq 0, t \in I, i=1,2, \cdots, m \\
z(t) \in K, \omega^{j}(t) \in C^{j}, j=1,2, \cdots, m
\end{gathered}
$$

We review some well known facts about a support function for easy reference. Let $\Gamma$ be a compact convex set in $R^{n}$. Then the support function of $\Gamma$ denoted by $s(x(t) \mid \Gamma)$ is defined as

$$
s(x(t) \mid \Gamma)=\max \left\{x(t)^{\mathrm{T}} v(t): v(t) \in \Gamma, t \in I\right\}
$$

A support function, being convex and everywhere finite, has a subdifferential in the sense of convex analysis, that is, there exists $z: I \rightarrow R^{n}$ such that $s(y(t) \mid \Gamma) \geq s(x(t) \mid \Gamma)+z(t)^{\mathrm{T}}(y(t)-x(t))$ for all $x$. The subdifferential of $s(x(t) \mid \Gamma)$ is given by $s(x(t) \mid \Gamma)=\left\{z(t) \in \Gamma: z(t)^{\mathrm{T}} x(t)=S(x(t) \mid \Gamma)\right\}$. Let $N_{\Gamma}(x(t))$ be normal cone at a point $x(t) \in \Gamma$. Then $y(t) \in N_{\Gamma}(x)$ if and only if $s(y(t) \mid \Gamma)=x(t)^{\mathrm{T}} y(t)$ or, equivalently, $x(t)$ is in the subdifferential of $s$ at $y(t)$.

In order to relax the pseudoconvexity in [1], Mond-Weir type dual to (CP) is constructed and various duality theorems are derived. Particular cases are deduced and it is also indicated that our results can be considered as the dynamic generalization of the duality results for nonlinear programming problem with support functions.

\section{Mond-Weir Type Duality}

We propose the following Mond-Weir type dual (M-WCD) to the control problem (CP):

Dual (M-WCD): Maximize $\int_{I}\left\{f(t, x, u)+u^{\mathrm{T}}(t) z(t)\right\} \mathrm{d} t$

subject to

$$
\begin{gathered}
x(a)=0=x(b) \\
f_{x}(t, x, u)+\sum_{j=1}^{m} \lambda^{j}(t)\left(g_{x}^{j}(t, x, u)+x(t)^{\mathrm{T}} \omega^{i}(t)\right)+\mu(t)^{\mathrm{T}} h_{x}(t, x, u)=\dot{\mu}(t), t \in I
\end{gathered}
$$




$$
\begin{gathered}
f_{u}(t, x, u)+z+\lambda^{t} g_{u}(t, x, u)+\mu(t)^{\mathrm{T}} h_{u}(t, x, u)=\dot{\mu}(t), t \in I \\
\sum_{j=1}^{m} \lambda^{i} \int_{I}\left\{g^{j}(t, x, u)+x(t)^{\mathrm{T}} \omega^{j}\right\} \mathrm{d} t \geq 0 \\
\int_{I} \mu(t)^{\mathrm{T}}(h(t, x, u)-\dot{x}) \mathrm{d} t \geq 0 \\
\lambda^{j}(t) \geq 0 \quad t \in I, j=1,2, \cdots, m \\
z(t) \in K \\
\omega^{j}(t) \in C^{j}, j=1,2, \cdots, m
\end{gathered}
$$

Theorem 1. (Weak Duality): Assume that

$\left(\mathbf{A}_{1}\right):(\bar{x}, \bar{u})$ is feasible for (CP),

$\left(\mathbf{A}_{2}\right):\left(x, u, \lambda^{1}, \cdots, \lambda^{m}, \mu, z, \omega^{1}, \cdots, \omega^{m}\right)$ is feasible for the problem (M-WCD),

$\left(\mathbf{A}_{3}\right): \int_{I}\{f(t, \cdot, \cdot)+(\cdot) z(t)\} \mathrm{d} t$, for $z(t) \in K$ is pseudoconvex, and

$\left(\mathbf{A}_{4}\right): \sum_{j=1}^{m} \int_{I} \lambda^{i}(t)\left(g^{j}(t, \cdot, \cdot)+(\cdot)^{\mathrm{T}} \omega^{j}(t)\right) \mathrm{d} t$ for all $\omega^{j}(t) \in C^{j}, j=1,2, \cdots, m$ and $\int_{I} \mu^{\mathrm{T}}(t)(h(t, \cdot, \cdot)-x(\cdot)) \mathrm{d} t$ are quasiconvex at $(x, u)$.

Then

$$
\inf (\mathrm{CP}) \geq \sup (\mathrm{CD})
$$

Proof: Since $\lambda^{i}(t) \geq 0, j=1,2, \cdots, m_{j}, t \in I, g^{i}(t, \bar{x}, \bar{u})+s\left(\bar{x}(t) \mid C^{j}\right), h(t, \bar{x}, \bar{u})-\bar{x}(t)=0, t \in I$, we have

$$
\int_{I}\left[\sum_{j=1}^{m} \lambda^{j}(t)\left(g^{i}(t, \bar{x}, \bar{u})+\bar{x}(t)^{\mathrm{T}} \omega^{j}(t)\right)\right] \mathrm{d} t \leq 0
$$

and

$$
\int_{I} \mu(t)^{\mathrm{T}}(h(t, \bar{x}, \bar{u})-\dot{\bar{x}}) \mathrm{d} t \leq 0
$$

Combining these inequalities with (14) and (15) respectively, we have

$$
\int_{I}\left[\sum_{j=1}^{m} \lambda^{j}(t)\left(g^{i}(t, \bar{x}, \bar{u})+\bar{x}(t)^{\mathrm{T}} \omega^{j}(t)\right)\right] \mathrm{d} t \leq \int_{I}\left[\sum_{j=1}^{m} \lambda^{j}(t)\left(g^{i}(t, x, u)+x(t)^{\mathrm{T}} \omega^{j}(t)\right)\right] \mathrm{d} t
$$

and

$$
\int_{I} \mu(t)^{\mathrm{T}}(h(t, \bar{x}, \bar{u})-\dot{\bar{x}}) \mathrm{d} t \leq \int_{I} \mu(t)^{\mathrm{T}}(h(t, \bar{x}, \bar{u})-\dot{x}) \mathrm{d} t
$$

These, because of the hypothesis $\left(\mathrm{A}_{4}\right)$ yields

$$
\begin{gathered}
\int_{I}\left\{(\bar{x}-x)^{\mathrm{T}} \sum_{j=1}^{m} \lambda_{t}^{j}\left(g_{x}^{j}(t, x, u)+\omega^{j}(t)\right)+(\bar{u}-u)^{\mathrm{T}} \lambda^{\mathrm{T}} g_{x}(t, x, u)\right\} \leq 0 \\
\int_{I}\left\{(\bar{x}-x)^{\mathrm{T}}\left(\mu(t)^{\mathrm{T}} h_{x}(t, x, u)+\dot{\mu}(t)\right)+(\bar{x}-u)^{\mathrm{T}} \mu(t)^{\mathrm{T}} h_{u}(t, x, u)\right\} \mathrm{d} t \leq 0
\end{gathered}
$$

Combining (19) and (20) and then using (12) and (13), we have

$$
\int_{I}\left\{(\bar{x}-x)^{\mathrm{T}}\left(f_{x}(t, x, u)+(\bar{u}-u)^{\mathrm{T}} z\right)\right\} \geq 0
$$


This, due to the pseudoconvexity of $\int_{I}\left(f(t, . .)+.(.)^{\mathrm{T}} z\right) \mathrm{d} t$ for $z(t) \in K$ implies

$$
\int_{I}\left(f(t, \bar{x}, \bar{u})+\bar{u}^{\mathrm{T}} z\right) \mathrm{d} t \geq \int_{I}\left(f(t, x, u)+u^{\mathrm{T}} z\right) \mathrm{d} t
$$

Since $\bar{u}(t)^{\mathrm{T}} z(t) \leq S(\bar{x}(t) \mid K)$, the above inequality gives

$$
\int_{I}\{f(t, \bar{x}, \bar{u})+\leq(\bar{u}(t) \mid K)\} \geq \int_{I}\left\{f(t, x, u)+u(t)^{\mathrm{T}} z(t)\right\} \mathrm{d} t
$$

yielding

$$
\inf (\mathrm{CP}) \geq \sup (\mathrm{CD})
$$

Theorem 2. (Strong Duality): If $(\bar{x}, \bar{u})$ is an optimal solution of (CP) and is normal, then there exist piecewise smooth $\lambda: I \rightarrow R^{m}$ with $\lambda=\left(\lambda_{1}, \lambda_{2}, \cdots, \lambda_{m}\right), \quad z: I \rightarrow R^{n}$ and such that $\left(\bar{x}, \bar{u}, \bar{\lambda}^{1}, \cdots, \bar{\lambda}^{m}, \bar{z}, \bar{\omega}^{1}, \bar{\omega}^{2}, \cdots, \bar{\omega}^{m}\right)$ is feasible for (M-WCD) and the corresponding values of (CP) and (M-WCD) are equal. If also, the hypotheses of Theorem 1 hold, then $\left(\bar{x}, \bar{u}, \bar{\lambda}^{1}, \cdots, \bar{\lambda}^{m}, \bar{z}, \bar{\omega}^{1}, \bar{\omega}^{2}, \cdots, \bar{\omega}^{m}\right)$ is optimal solution of the problem (M-WCD).

Proof: Since $(\bar{x}, \bar{u})$ is an optimal solution of (CP) and is normal, it follows by Proposition 2 that there exist piecewise smooth $\lambda^{j}: I \rightarrow R^{m}, \quad j=1,2, \cdots, m, \quad \mu: I \rightarrow R^{n}, \quad z: I \rightarrow R^{n}$ and $\omega^{j}: I \rightarrow R^{n}(j=1,2, \cdots, m)$. satisfying for all $t \in I$, the conditions (4)-(10) are satisfied. The conditions (4)-(6) together with (9) and (10) imply that $\left(\bar{x}, \bar{u}, \bar{\lambda}^{1}, \cdots, \bar{\lambda}^{m}, \bar{z}, \bar{\omega}^{1}, \bar{\omega}^{2}, \cdots, \bar{\omega}^{m} ; \bar{\mu}\right)$ is feasible for (M-WCD). Using $\bar{x}(t)^{T} z(t)=s(x(t) \mid K)$, we obtain,

$$
\int_{I}(f(t, \bar{x}, \bar{u})+T+S(\bar{x}(t) \mid K))=\int_{I}\left(f(t, \bar{x}, \bar{u})+\bar{x}(t)^{\mathrm{T}} z(t)\right) \mathrm{d} t
$$

The equality of the objective functionals of the problems (CP) and (M-WCD) follows. This along with the hypotheses of Theorem 1 , the optimality of $\left(\bar{x}, \bar{u}, \bar{\lambda}^{1}, \cdots, \bar{\lambda}^{m}, \bar{z}, \bar{\omega}^{1}, \bar{\omega}^{2}, \cdots, \bar{\omega}^{m}\right)$ for (M-WCD) follows.

The following gives the Mangasarian type strict converse duality theorem:

Theorem 3. (Strict Converse Duality): Assume that

$\left(\mathbf{A}_{1}\right):(\bar{x}, \bar{u})$ is an optimality solution of (CP) and is normal;

$\left.\mathbf{( A}_{2}\right):\left(\hat{x}, \hat{u}, \hat{\lambda}^{1}, \cdots, \hat{\lambda}^{m}, \hat{\mu}, \hat{z}, \hat{\omega}^{1}, \cdots, \hat{\omega}^{m}\right)$ is an optimal solution of (M-WCD),

$\left(\mathbf{A}_{3}\right): \int_{I}\left\{f(t, \cdot, \cdot)+(\cdot)^{\mathrm{T}} z(t)\right\} \mathrm{d} t$ in strictly is pseudoconvex for all $z(t) \in K$, and

$\left(\mathbf{A}_{4}\right): \sum_{j=1}^{m} \int_{I}\left(g^{j}(t, \cdot, \cdot)+(\cdot)^{\mathrm{T}} \omega^{j}(t)\right) \mathrm{d} t \quad$ for $\quad$ all $\quad \omega^{j}(t) \in C^{j}, j=1,2, \cdots, m \quad$ and $\quad \int_{I} \mu^{\mathrm{T}}(t)(h(t, \cdot, \cdot)-\dot{x}) \mathrm{d} t \quad$ are quasi convex.

Then $(\bar{x}, \bar{u})=(\hat{x}, \hat{u})$, i.e. $(\hat{\bar{x}}, \hat{\bar{u}})$ is an optimal solution of $(\mathrm{CP})$.

Proof: Assume that $(\bar{x}, \bar{u}) \neq(\hat{x}, \hat{u})$ and exhibit a contradiction. Since $(\bar{x}, \bar{u})$ is an optimality solution of (CP). By Theorem 2 there exist $\left(\bar{\lambda}, \bar{\mu}, \bar{z}, \bar{\omega}^{1}, \cdots, \bar{\omega}^{m}\right)$ with $\bar{\lambda}=\left(\bar{\lambda}^{1}, \bar{\lambda}^{2}, \cdots, \bar{\lambda}^{m}\right)$ such that $\left(\bar{x}, \bar{u}, \bar{\lambda}^{1}, \cdots, \bar{\lambda}^{m}, \bar{z}, \bar{\omega}^{1}, \cdots, \bar{\omega}^{m}\right)$ is an optimal solution of (M-WCD).

Thus

$$
\int_{I}\left\{f(t, \bar{x}, \bar{u})+\bar{u}(t)^{\mathrm{T}} \bar{z}(t)\right\} \mathrm{d} t=\int_{I}\{f(t, \hat{x}, \hat{u})+\hat{u}(t) \hat{z}(t)\} \mathrm{d} t
$$

Since $(\bar{x}, \bar{u})$ is feasible for (CP) and $\left(\hat{x}, \hat{u}, \hat{\lambda}^{1}, \cdots, \hat{\lambda}^{m}, \hat{\mu}, \hat{z}, \hat{\omega}^{1}, \cdots, \hat{\omega}^{m}\right)$ for (M-WCD), we have

and

$$
\sum_{i=1}^{m} \int_{I} \hat{\lambda}^{i}(t)\left(g^{i}(t, \bar{x}, \bar{u})+\bar{x}(t)^{\mathrm{T}} \omega^{i}(t)\right) \mathrm{d} t \leq \sum_{i=1}^{m} \int_{I} \hat{\lambda}^{i}(t)\left(g^{i}(t, \hat{x}, \hat{u})+\hat{x}(t)^{\mathrm{T}} \hat{\omega}^{i}(t)\right) \mathrm{d} t
$$

$$
\int_{I} \hat{\mu}(t)(h(t, \bar{x}, \bar{u})-\dot{\bar{x}}(t)) \mathrm{d} t \leq \int_{I} \hat{\mu}(t)(h(t, \hat{x}, \hat{u})-\dot{\hat{x}}(t)) \mathrm{d} t
$$


These, because of the hypothesis (A4) imply the merged inequality

$$
\begin{aligned}
& \int_{I}\left\{(\bar{x}-\hat{x})^{\mathrm{T}}\left(\sum_{j=1}^{m} \hat{\lambda}^{i}(t)\left(g_{x}^{j}(t, \hat{x}, \hat{u})+\hat{\omega}^{i}(t)\right)+\left(\hat{\mu}(t)^{\mathrm{T}} h_{x}(t, \hat{x}, \hat{u})-\dot{\hat{\mu}}(t)\right)\right)\right. \\
& \left.+(\bar{u}-\hat{u})^{\mathrm{T}}\left(\sum_{j=1}^{m} \hat{\lambda}(t)^{\mathrm{T}} g_{\hat{u}}(t, \hat{x}, \hat{u})+\hat{\mu}(t) h_{u}(t, \hat{x}, \hat{u})\right)\right\} \mathrm{d} t \leq 0
\end{aligned}
$$

This, by using the equality constraints (12) and (13) of (M-WCD) gives

$$
\int_{I}\left[(\bar{x}-\hat{x})^{\mathrm{T}} f_{x}(t, \hat{x}, \hat{u})+(\bar{u}-\hat{u})^{\mathrm{T}}\left(f_{u}(t, \hat{x}, \hat{u})+\hat{z}(t)\right)\right] \mathrm{d} t \geq 0
$$

By the hypothesis $\left(A_{2}\right)$, this implies

$$
\int_{I}\left(f(t, \bar{x}, \bar{u})+\bar{\mu}^{\mathrm{T}} \hat{z}(t)\right) \mathrm{d} t>\int_{I}(f(t, \hat{x}, \hat{u})+\hat{\mu}(t) \hat{z}(t)) \mathrm{d} t=\int_{I}\left(f(t, \bar{x}, \bar{u})+\bar{\mu}(t)^{\mathrm{T}} \hat{z}(t)\right) \mathrm{d} t
$$

(using (21)). Consequently, we have

$$
\int_{I} \bar{u}(t)^{\mathrm{T}} \hat{z}(t) \mathrm{d} t>\int_{I} \bar{u}(t)^{\mathrm{T}} \bar{z}(t) \mathrm{d} t
$$

Since $\bar{u}(t) \bar{z}(t)=S(\bar{u}(t) \mid K)$ for $\bar{z}(t) \in K$ and $\bar{u}(t)^{\mathrm{T}} \hat{z}(t) \leq s(\bar{u}(t) \mid K)$ for $\hat{z}(t) \in K$, this yields,

$$
\int_{I} s\left(\bar{u}(t)^{\mathrm{T}} \bar{Z}(t)\right) \mathrm{d} t>\int_{I} s(\bar{u}(t) \mid K) \mathrm{d} t
$$

This cannot happen. Hence $(\bar{x}, \bar{u})=(\hat{x}, \hat{u})$.

\section{Converse Duality}

The problem (M-WCD) can be written as the follows:

Maximize: $\psi\left(x, \mu, z, \lambda^{1}, \cdots, \lambda^{m}, \omega^{1}, \cdots, \omega^{m}\right)$

subject to

$$
\begin{gathered}
x(a)=0, x(b)=0 \\
\theta^{1}\left(t, x(t) u(t), \lambda^{1}(t), \cdots, \lambda^{m}(t), \mu(t), \omega^{1}(t), \omega^{2}(t), \cdots, \omega^{m}(t)\right)=0, t \in I \\
\theta^{2}\left(t, x(t) u(t), z(t), \lambda^{1}(t), \cdots, \lambda^{m}(t), \mu(t), \omega^{1}(t), \omega^{2}(t), \cdots, \omega^{m}(t)\right)=0, t \in I \\
\int_{I} \sum_{j=1}^{m} \lambda^{j}(t)\left(g^{j}(t, x, u)+x(t)^{\mathrm{T}} \omega^{j}(t)\right) \mathrm{d} t \geq 0 \\
\int_{I} \mu(t)^{\mathrm{T}}(h(t, x, u)-\dot{x}(t)) \mathrm{d} t \geq 0 \\
z(t) \in K, \quad t \in I \\
\omega^{j}(t) \in C^{j}, t \in I, j=1,2, \cdots, m \\
\lambda(t) \geq 0, t \in I, j=1,2, \cdots, m
\end{gathered}
$$

where

$$
\begin{gathered}
\theta^{1}=\theta^{2}(t, x, u, z, \lambda, \mu)=f_{x}+\sum_{j=1}^{m} \lambda^{j}(t)\left(g_{x}^{j}+\omega^{j}(t)\right)+\mu(t)^{\mathrm{T}} h_{x}+\dot{\mu}(t) \\
\theta^{2}=\theta^{2}(t, x, u, z, \lambda, \mu)=f_{u}+z+\lambda^{\mathrm{T}} g_{x}+\mu^{\mathrm{T}} h_{u}
\end{gathered}
$$

Consider $\theta^{1}\left(., x(),. u(),. \lambda(),. \omega_{2}^{1}(),. \cdots, \omega^{m}(),. \mu().\right)$ and $\theta^{2}(t, x(),. u(),. \lambda(),. z(.) \mu()$.$) as defining a map-$ 
pings $Q^{1}: X \times u \times \Lambda \times W^{1} \times W^{2} \times \cdots \times W^{m} \times V \rightarrow B^{1}$ and $Q^{2}: X \times u \times Z \times \Lambda \times V \rightarrow B^{2}$ respectively where $\Lambda$ is the space of piecewise smooth $\lambda, V$ is space of piececewise smooth $\mu, W^{j}$ is the space of piecewise of smooth $W^{j}, \quad j=1,2, \cdots, m, B^{1}$ and $B^{2}$ are Banach spaces. $\theta^{1}=\left(x, u, \lambda, \mu, \omega^{1}, \omega^{2}, \cdots, \omega^{m}\right)$ and $\theta^{2}=(x, u, \lambda, \mu, z)$ with $\lambda=\left(\lambda^{1}, \cdots, \lambda^{m}\right)$. Here some restrictions are required on the equality constraints. For this, it suffices that if the Fre'che't derivatives $Q^{1^{\prime}}=\left(\theta_{x}^{1}(), \theta_{u}^{1}(), \theta_{\lambda}^{1}(), \theta_{\mu}^{1}(), \theta_{\omega^{1}}^{1}(), \cdots, \theta_{\omega^{m}}^{1}()\right)$ and

$Q^{2^{\prime}}=\left(\theta_{x}^{2}(), \theta_{u}^{2}(), \theta_{\lambda}^{2}(), \theta_{\mu}^{2}(), \theta_{\omega^{1}}^{2}(), \cdots, \theta_{\omega^{m}}^{2}()\right)$, have weak * closed range.

Theorem 4. (Converse Duality): Assume that

$\left(\mathbf{A}_{\mathbf{1}}\right): \quad f, g$ and $h$ are twice continuously differentiable.

$\left(\mathbf{A}_{2}\right):\left(x, u, \lambda, \mu, \mathrm{z}, \omega^{1}, \cdots, \omega^{m}\right)$ is an optimal solution of (CP).

$\left(\mathbf{A}_{3}\right): Q^{1^{\prime}}$ and $Q^{2^{\prime}}$ have weak * closed ranges.

$\left(\mathbf{A}_{4}\right): \int_{I} \sigma(t)^{\mathrm{T}} A, \sigma(t) \mathrm{d} t=0 \Rightarrow \sigma(t)=0, \quad t \in I$ for some $\sigma(t) \in R^{n}$, and

$$
A=\left(\begin{array}{ll}
f_{x x}+\lambda^{\mathrm{T}}(t) g_{x x}+\mu(t)^{\mathrm{T}} h_{x x} & f_{u x}+\lambda^{\mathrm{T}}(t) g_{u x}+\mu^{\mathrm{T}}(t) h_{u x} \\
f_{u x}+\lambda^{\mathrm{T}}(t) g_{u x}+\mu(t)^{\mathrm{T}} h_{u x} & f_{u u}+\lambda^{\mathrm{T}}(t) g_{u u}+\mu(t)^{\mathrm{T}} h_{u u}
\end{array}\right)
$$

(A): 1) The gradient vectors $\sum_{i=1}^{m} \lambda^{i}(t)\left(g_{x}^{i}+\omega^{i}(t)\right)$ and $\lambda(t)^{\mathrm{T}}$ are linearly independent, or

2) The gradient vectors $\mu(t)^{\mathrm{T}} h_{x}+\dot{\mu}(t)$ and $\mu(t)^{t} h_{u}$ are linearly independent.

(A): $\mu(a)=0=\mu(b)$.

Proof: Since $\left(x, u, \lambda^{1}, \cdots, \lambda^{m}, \mu, z, \omega^{1}, \cdots, \omega^{m}\right)$ is an optimal solution of (M-WCD), by Proposition 1 there exists $\alpha_{0} \in R, \quad \gamma \in R$, and $\beta \in R$, and piecewise smooth functions $\phi^{1}: I \rightarrow R^{n}, \phi^{2}: I \rightarrow R^{m}, \quad \delta^{i}: I \rightarrow R$, $i=1,2, \cdots, m$, such that

$$
\begin{gathered}
\alpha_{0} f_{x}+\phi^{1}(t)^{\mathrm{T}}\left(f_{x x}+\lambda(t)^{\mathrm{T}} g_{x x}+\mu(t)^{\mathrm{T}} h_{x x}\right)+\phi^{2}(t)^{\mathrm{T}}\left(f_{u x}+\lambda(t)^{\mathrm{T}} g_{u x}+\mu(t)^{\mathrm{T}} h_{u x}\right) \\
+\gamma\left(\sum_{i=1}^{m} \lambda^{i}(t)\left(g_{x}^{i}+\omega^{i}(t)\right)\right)+\beta\left(\mu(t)^{\mathrm{T}} h_{x}-\dot{\mu}(t)\right)=0, t \in I \\
\alpha_{0}\left(f_{u}+z\right)+\phi^{1}(t)\left(f_{x u}+\lambda^{\mathrm{T}}(t) g_{x u}+\mu^{\mathrm{T}}(t) h_{x u}\right)+\phi^{2}(t)\left(f_{x u}+\lambda^{\mathrm{T}}(t) g_{x u}+\mu^{\mathrm{T}}(t) h_{x u}\right) \\
+\gamma\left(\left(\lambda(t)^{\mathrm{T}} g_{x}\right)+\beta\left(\mu(t)^{\mathrm{T}} h_{u}\right)\right)=0, t \in I \\
\phi^{1}(t)\left(g_{x}^{i}+\omega^{i}(t)\right)+\phi^{2}(t) g_{u}^{i}+\gamma\left(g^{i}+x(t)^{\mathrm{T}} \omega^{i}(t)\right)+\delta^{i}(t)=0, t \in I \\
\phi^{1}(t) h_{x}-\dot{\phi}^{1}(t)+\phi^{2}(t) h_{u}+\beta(h-\dot{x})=0 \\
\alpha_{0} \bar{u}(t)+\phi^{2}(t) \in-N_{K}(z), t \in I \\
\phi^{1} \lambda^{i}(t)+\gamma \lambda^{i}(t) \bar{x}(t) \in-N_{C^{i}}\left(\omega^{i}\right), i=1,2, \cdots, m, t \in I \\
\gamma \iint_{I}\left(\sum \lambda^{i}(t)\left(g^{i}+x(t)^{\mathrm{T}} \omega^{i}(t)\right)\right) \mathrm{d} t=0 \\
\beta \int \mu(t)^{\mathrm{T}}(h-\dot{\bar{x}}) \mathrm{d} t=0 \\
\delta(t)^{\mathrm{T}} \lambda(t)=0, t \in I \\
\left(\alpha_{0}, \gamma, \beta, \delta(t)\right) \geq 0 \\
\left(\alpha_{0}, \phi^{1}(t), \phi^{2}(t), \gamma, \beta, \delta(t)\right) \neq 0, t \in I
\end{gathered}
$$


Multiplying (24) by $\lambda^{i}(t), t \in I, i=1,2, \cdots, m$ and summing over $i$ and then integrating using (28), we have

$$
\int_{I}\left\{\phi^{1}(t)^{\mathrm{T}}\left(\sum_{i=1}^{m} \lambda^{i}(t)\left(g_{x}^{i}+\omega^{i}(t)\right)\right)+\phi^{2}(t)^{\mathrm{T}}\left(\lambda(t)^{\mathrm{T}} g_{x}\right)\right\} \mathrm{d} t=0, t \in I
$$

which can be written as,

$$
\int_{I}\left(\phi^{1}(t)^{\mathrm{T}}, \phi^{2}(t)^{\mathrm{T}}\right)\left(\begin{array}{c}
\sum_{i=1}^{m}\left(\lambda^{i}(t)\left(g_{x}^{i}+\omega^{i}(t)\right)\right) \\
\lambda(t)^{\mathrm{T}} g_{x}
\end{array}\right) \mathrm{d} t=0
$$

Multiplying (25) by $\mu(t)$ and then integrating and using (29), we have

$$
\int_{I}\left(\phi^{1}(t) \mu(t)^{\mathrm{T}} h_{x}+\phi^{2}(t) \mu^{\mathrm{T}}(t) h_{u}\right) \mathrm{d} t-\left.\mu(t) \phi^{1}(t)\right|_{t=a} ^{t=b}+\int_{I} \dot{\mu}(t) \phi^{1}(t) \mathrm{d} t=0
$$

This implies

$$
\int_{I}\left(\phi^{1}(t)^{\mathrm{T}}\left(\mu(t)^{\mathrm{T}} h_{x}-\dot{\mu}(t)\right)+\phi^{2}(t)^{\mathrm{T}}\left(\mu(t)^{\mathrm{T}} h_{u}\right)\right) \mathrm{d} t=0
$$

or

$$
\int_{I}\left(\phi^{1}(t)^{\mathrm{T}}, \phi^{2}(t)^{\mathrm{T}}\right)\left(\begin{array}{c}
\mu(t)^{\mathrm{T}} h_{x}+\dot{\mu}(t) \\
\mu(t)^{\mathrm{T}} h_{u}
\end{array}\right) \mathrm{d} t=0
$$

Using the equality constraints (12) and (13) of the problem (M-WCD) in (22) and (23), we have

$$
\begin{aligned}
& \left(\gamma-\alpha_{0}\right) \sum_{i=1}^{m} \lambda^{i}(t)\left(g_{x}^{i}+\omega^{j}\right)+\left(\beta-\alpha_{0}\right)\left(\mu(t)^{\mathrm{T}} h_{x}+\dot{\mu}(t)\right) \\
& +\phi^{1}(t)\left(f_{x x}+\lambda(t)^{\mathrm{T}} g_{x x}+\mu(t)^{\mathrm{T}} h_{x x}\right)+\phi^{2}(t)\left(f_{u x}+\lambda(t)^{\mathrm{T}} g_{u x}+\mu(t)^{\mathrm{T}} h_{u x}\right)=0 \\
& \left(\gamma-\alpha_{0}\right)\left(\lambda(t)^{\mathrm{T}} g_{x}\right)+\left(\beta-\alpha_{0}\right)\left(\mu(t)^{\mathrm{T}} h_{u}\right)+\phi^{1}(t)\left(f_{x u}+\lambda(t)^{\mathrm{T}} g_{x u}+\mu(t)^{\mathrm{T}} h_{x u}\right) \\
& +\phi^{2}(t)\left(f_{u u}+\lambda(t)^{\mathrm{T}} g_{u u}+\mu(t)^{\mathrm{T}} h_{u u}\right)=0
\end{aligned}
$$

Combining (35) and (36), we have

$$
\begin{aligned}
& \left(\gamma-\alpha_{0}\right)\left(\begin{array}{c}
\sum_{i=1}^{m} \lambda^{i}(t)\left(g_{x}^{i}+\omega^{i}\right) \\
\lambda(t)^{\mathrm{T}} g_{u}
\end{array}\right)+\left(\beta-\alpha_{0}\right)\left(\begin{array}{c}
\mu(t)^{\mathrm{T}} h_{x}+\dot{\mu}(t) \\
\mu(t)^{\mathrm{T}} h_{u}
\end{array}\right) \\
& +\left(\begin{array}{cc}
f_{x x}+\lambda^{\mathrm{T}}(t)^{\mathrm{T}} g_{x x}+\mu(t)^{\mathrm{T}} h_{x x} & f_{u x}+\lambda^{\mathrm{T}}(t) g_{u x}+u(t)^{\mathrm{T}} h_{u x} \\
f_{x u}+\lambda^{\mathrm{T}}(t) g_{x u}+u(t)^{\mathrm{T}} h_{x u} & f_{u u}+\lambda^{\mathrm{T}}(t) g_{u u}+u(t)^{\mathrm{T}} h_{u u}
\end{array}\right)\left(\begin{array}{c}
\phi^{1}(t) \\
\phi^{2}(t)
\end{array}\right) \mathrm{d} t=0
\end{aligned}
$$

This by premultiplying by $\left(\phi^{1}(t)^{\mathrm{T}}, \phi^{2}(t)^{\mathrm{T}}\right)$, and then integrating, we have

$$
\begin{aligned}
& \left(\gamma-\alpha_{0}\right) \int_{I}\left(\phi^{1}(t)^{\mathrm{T}}, \phi^{2}(t)^{\mathrm{T}}\right)\left(\begin{array}{c}
\sum_{i=1}^{m} \lambda^{i}(t)\left(g_{x}^{i}+\omega^{i}(t)\right) \\
\lambda(t)^{\mathrm{T}} g_{u}
\end{array}\right) \mathrm{d} t+\left(\beta-\alpha_{0}\right) \int_{I}\left(\phi^{1}(t)^{\mathrm{T}}, \phi^{2}(t)^{\mathrm{T}}\right)\left(\begin{array}{c}
\mu(t)^{\mathrm{T}} h_{x}+\dot{\mu}(t) \\
\lambda(t)^{\mathrm{T}} h_{u}
\end{array}\right) \mathrm{d} t \\
& +\int_{I}\left(\phi^{1}(t)^{\mathrm{T}}, \phi^{2}(t)^{\mathrm{T}}\right)\left(\begin{array}{cc}
f_{x x}+\lambda^{\mathrm{T}}(t)^{\mathrm{T}} g_{x x}+\mu(t)^{\mathrm{T}} h_{x x} & f_{u x}+\lambda^{\mathrm{T}}(t) g_{u x}+u(t)^{\mathrm{T}} h_{u x} \\
f_{x u}+\lambda^{\mathrm{T}}(t) g_{x u}+u(t)^{\mathrm{T}} h_{x u} & f_{u u}+\lambda^{\mathrm{T}}(t) g_{u u}+u(t)^{\mathrm{T}} h_{u u}
\end{array}\right)\left(\begin{array}{l}
\phi^{1}(t) \\
\phi^{2}(t)
\end{array}\right) \mathrm{d} t=0
\end{aligned}
$$

Using (33) and (34), we have 


$$
\int_{I}\left(\phi^{1}(t)^{\mathrm{T}}, \phi^{2}(t)^{\mathrm{T}}\right)\left(\begin{array}{cc}
f_{x x}+\lambda^{\mathrm{T}}(t)^{\mathrm{T}} g_{x x}+\mu(t)^{\mathrm{T}} h_{x x} & f_{u x}+\lambda^{\mathrm{T}}(t) g_{u x}+u(t)^{\mathrm{T}} h_{u x} \\
f_{x u}+\lambda^{\mathrm{T}}(t) g_{x u}+u(t)^{\mathrm{T}} h_{x u} & f_{u u}+\lambda^{\mathrm{T}}(t) g_{u u}+u(t)^{\mathrm{T}} h_{u u}
\end{array}\right)\left(\begin{array}{c}
\phi^{1}(t) \\
\phi^{2}(t)
\end{array}\right) \mathrm{d} t=0
$$

This because of hypothesis $\left(\mathrm{A}_{4}\right)$ implies

$$
\phi^{1}(t)=0=\phi^{2}(t), t \in I
$$

Using $\phi^{1}(t)=0=\phi^{2}(t), t \in I$, gives

$$
\left(\gamma-\alpha_{0}\right) \sum_{i=1}^{m} \lambda^{i}(t)\left(g_{x}^{i}+\omega^{i}(t)\right)+\left(\beta-\alpha_{0}\right)\left(\mu(t) h_{x}+\dot{\mu}(t)\right)=0, t \in I
$$

This, because of hypothesis $\left(A_{5}\right)$ implies

$$
\left(\gamma-\alpha_{0}\right)=0,\left(\beta-\alpha_{0}\right)=0
$$

Assume $\alpha_{0}=0$, (37) gives $\gamma=0=\beta$. from (24) it follows $\delta(t)=0, t \in I$. Consequently we have

$$
\left(\alpha_{0}, \phi^{1}(t), \phi^{2}(t), \gamma, \beta, \delta(t)\right)=0, t \in I,
$$

contradicting (32). Hence $\alpha_{0}>0, \gamma>0$ and $\beta>0$. The relations (26) and (27) gives

$$
\bar{u}(t) \in-N_{K}(z) \text { and } \bar{x}(t) \in-N_{C^{i}}\left(\omega^{i}\right), i=1,2, \cdots, m
$$

yielding $\bar{u}(t)^{\mathrm{T}} z(t)=s(\bar{u}(t) \mid K)$ and $\bar{x}(t)^{\mathrm{T}} \omega^{i}(t)=S\left(\bar{x}(t) \mid C^{i}\right), i=1,2, \cdots, m$.

From (24), we have

$$
g^{i}+\bar{x}(t)^{\mathrm{T}} \omega^{i}(t) \leq 0, i=1,2, \cdots, m, t \in I
$$

and

$$
\sum_{i=1}^{m} \lambda^{i}(t)\left(g^{i}+\bar{x}(t)^{\mathrm{T}} \omega^{i}(t)\right)=0
$$

From (25), we have

$$
h-\dot{\bar{x}}=0, t \in I
$$

and

$$
\bar{\mu}(t)^{\mathrm{T}}(h-\dot{\bar{x}})=0, t \in I
$$

The feasibility of $(\bar{x}, \bar{u})$ for (CP) follows from (38) and (40).

Consider

$$
\begin{aligned}
& \int_{I}\left(f(t, \bar{x}, \bar{u})+u(t)^{\mathrm{T}} z+\sum_{i \in I_{0}} \bar{\lambda}^{i}(t)\left(g^{i}(t, x, u)+x(t)^{\mathrm{T}} \omega^{i}(t)\right)+\bar{\mu}(t)^{\mathrm{T}}(h(t, \bar{x}, \bar{u})-\dot{\bar{x}})\right) \mathrm{d} t \\
& =\int_{I}(f(t, \bar{x}, \bar{u})+S(u(t) \mid K)) \mathrm{d} t
\end{aligned}
$$

(by using $\bar{u}(t)^{\mathrm{T}} z(t)=s(\bar{u}(t) \mid K)$ along (39) and (41)).

This along with the generalized convexity hypotheses implies that $\left(\bar{x}, \bar{u}, \bar{z}, \bar{\lambda}^{1}, \cdots, \bar{\lambda}^{m}, \bar{\mu}, \bar{\omega}^{1}, \cdots, \bar{\omega}^{m}\right)$ is an optimal solution of (M-WCD).

\section{Special Cases}

Let for $t \in I, \quad B(t)$ and $D^{j}(t),(j=1,2, \cdots, m)$ be positive semidefinite matrices and continuous on $I$. Then

$$
\left(u(t)^{\mathrm{T}} B(t) u(t)\right)^{\frac{1}{2}}=s(u(t) \mid K)
$$

where

$$
K=\left\{B(t) z(t) \mid z(t)^{\mathrm{T}} B(t) z(t) \leq 1, t \in I\right\}
$$


and

$$
\left(x(t)^{\mathrm{T}} D^{j}(t) x(t)\right)^{\frac{1}{2}}=s\left(x(t) \mid C^{j}\right), j=1,2, \cdots, m
$$

where

$$
C^{j}=\left\{D(t) \omega^{j}(t) \mid \omega^{j}(t)^{\mathrm{T}} D^{j}(t) \omega^{j}(t) \leq 1, t \in I\right\}
$$

Replacing the support function by their corresponding square root of a quadratic form, we have:

Primal $\left(\mathbf{C P}_{\mathbf{0}}\right)$ : Minimize $\int_{I}\left(f(t, x, u)+\left(x(t)^{\mathrm{T}} B(t) x(t)\right)^{\frac{1}{2}}\right) \mathrm{d} t$

subject to

$$
\begin{gathered}
x(a)=0=x(b) \\
g^{j}(t, x, u)+\left(x(t)^{\mathrm{T}} D^{j}(t) x(t)\right)^{\frac{1}{2}} \leq 0, t \in I, j=1,2, \cdots, m \\
\dot{x}(t)=h(t, x, u), t \in I
\end{gathered}
$$

(M-WCD $\mathbf{D}_{\mathbf{0}}$ : Maximize $\int_{I}\left(f(t, x, u)+u(t)^{\mathrm{T}} B(t) z(t)\right) \mathrm{d} t$

subject to

$$
\begin{gathered}
x(a)=0=x(b) \\
f_{x}(t, x, u)+\sum_{j=1}^{m} \lambda^{j}\left(g_{x}^{j}(t, x, u)+D^{j}(t) \omega^{j}(t)\right)+\mu(t)^{\mathrm{T}} h_{x}(t, x, u)=\dot{\mu}(t), t \in I \\
\sum_{j=1}^{m} \int_{I} \lambda^{j}\left(g^{j}(t, x, u)+x(t)^{\mathrm{T}} D^{j}(t) \omega^{j}(t)\right) \mathrm{d} t \geq 0 \\
\int_{I} \mu(t)^{\mathrm{T}}(h(t, x, u)-\dot{x}(t)) \mathrm{d} t \geq 0 \\
\lambda^{j}(t) \geq 0 \\
z(t)^{\mathrm{T}} B(t) z(t) \leq 1, t \in I \\
\omega^{j}(t)^{\mathrm{T}} D^{j}(t) \omega^{j}(t) \leq 1, t \in I, j=1,2, \cdots, m
\end{gathered}
$$

The above pair of nondifferentiable dual control problem has not been explicitly reported in the literature but the duality amongst $\left(\mathrm{CP}_{0}\right)$ and $\left(\mathrm{M}-\mathrm{WCD}_{0}\right)$ readily follows on the lines of the analysis of the preceding section.

\section{Related Nonlinear Programming Problems}

If the time dependency of the problem (CP) and (M-WCD) is removed, then these problems reduce to the following problem (NP), its Mond-Weir dual (M-WND):

Primal ( $\left.\mathbf{N P}_{\mathbf{0}}\right)$ : Minimize $f(x, u)+S(u \mid K)$ subject to

$$
\begin{gathered}
g^{j}(x, u)+S\left(x \mid D^{j}\right) \leq 0, j=1,2, \cdots, m \\
h(x, u)=0
\end{gathered}
$$

Dual (M-WND $\mathbf{D}_{\mathbf{0}}$ : Maximize $f(x, u)+u^{\mathrm{T}} z$ subject to 


$$
\begin{gathered}
f_{x}(x, u)+\sum_{j=1}^{m} \lambda^{j}\left(g_{x}^{j}(x, u)+x^{\mathrm{T}} \omega^{j}(x, u)\right)+\mu^{\mathrm{T}} h_{x}(x, u)=0 \\
f_{u}(x, u)+z+\lambda^{\mathrm{T}} g_{u}(x, u)+\mu^{\mathrm{T}} h_{u}(x, u)=0 \\
\sum_{j=1}^{m} \lambda^{j}\left(g^{j}(x, u)+S\left(x \mid D^{j}\right)\right) \geq 0 \\
\mu^{\mathrm{T}} h(x, u) \geq 0 \\
\lambda^{j} \geq 0, j=1,2, \cdots, m \\
z \in K, \omega^{j} \in C^{j}, j=1,2, \cdots, m
\end{gathered}
$$

The above nonlinear programming problems with support functions do not appear in the literature. However, if $f(x, u)$ and $S(u \mid K)$ are replaced by $f(x)$ and $S(x \mid K)$ respectively in $\left(\mathrm{NP}_{0}\right)$, then problems reduced to following studied by Hussain et al. [4].

$\left(\mathbf{P}_{1}\right)$ : Minimize $f(x)+S(x \mid K)$ subject to

$$
g^{i}(x)+S\left(x \mid C^{j}\right) \leq 0, i=1,2, \cdots, m
$$

(M-WCD): Maximize $f(x)+x(t)^{\mathrm{T}} z$ subject to

$$
\begin{gathered}
f_{x}(x)+z+\sum_{i=1}^{m} \lambda^{i}\left(g_{x}^{i}(x)+\omega^{i}\right)+\mu^{\mathrm{T}} h_{x}(x)=0 \\
\lambda^{i} \geq 0, i=1,2, \cdots, m \\
z \in K, \omega^{i} \in C^{i}, i=1,2, \cdots, m
\end{gathered}
$$

\section{Conclusion}

Mond-Weir type duality for a control problem having support functions is studied under generalized convexity assumptions. Special cases are deduced. The linkage between the results of this research and those of nonlinear programming problem with support functions is indicated. The problem of this research can be revisited in multiobjective setting.

\section{References}

[1] Husain, I., Ahmad, A. and Shah, A.R. (2014) On a Control Problem with Support Functions. (Submitted for Publication)

[2] Craven, B.D. (1978) Mathematical Programming and Control Theory. Chapman and Hall, Landon. http://dx.doi.org/10.1007/978-94-009-5796-1

[3] Mond, B. and Hanson, M. (1968) Duality for Control Problem. SIAM Journal on Control, 6, 114-120. http://dx.doi.org/10.1137/0306009

[4] Husain, I., Abha and Jabeen, Z. (2002) On Nonlinear Programming with Support Function. Journal of Applied Mathematics and Computing, 10, 83-99. 
Scientific Research Publishing (SCIRP) is one of the largest Open Access journal publishers. It is currently publishing more than 200 open access, online, peer-reviewed journals covering a wide range of academic disciplines. SCIRP serves the worldwide academic communities and contributes to the progress and application of science with its publication.

Other selected journals from SCIRP are listed as below. Submit your manuscript to us via either submit@scirp.org or Online Submission Portal.
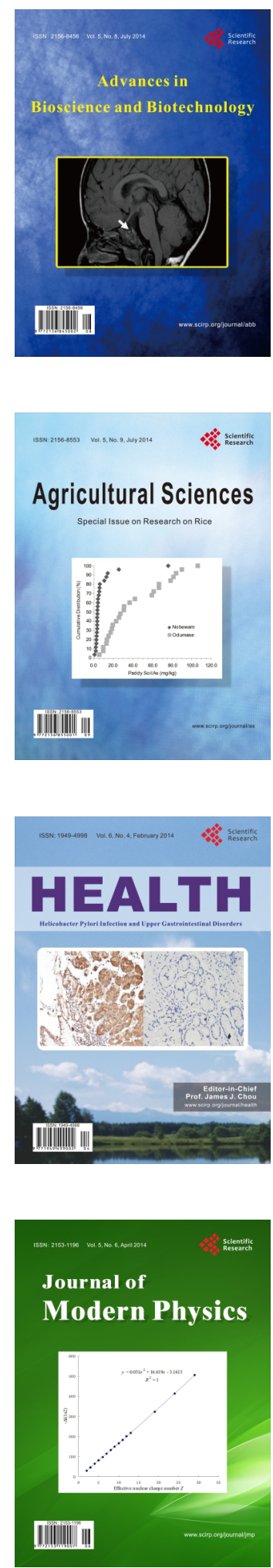
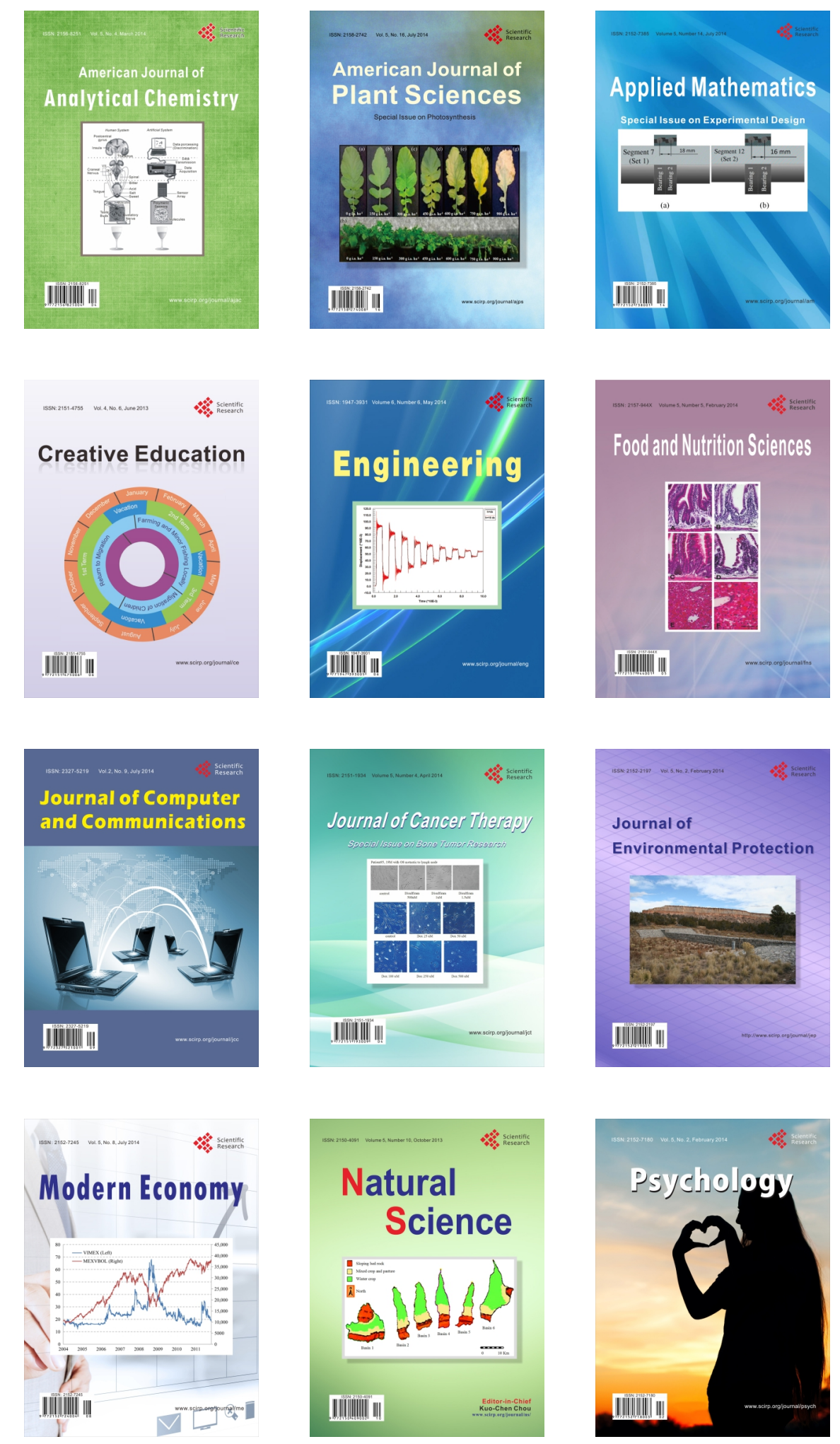